make such grand productions profitable. This publication would probably have a much greater sale among individual biologists and practising doctors if each article or at least each group of related articles could be bought separately, preferably in paperback form. That would have the further advaritage that the articles dealing with rapidly moving subjects, for example, immunological tolerance, could be rewritten at frequent intervals, so kecping the publication up to date.

The principal value of the work is likely to be as a source of authoritative information about particular topics which one may have to read up either to follow a line of ideas and to see how something has been worked out in an unfamiliar field, or when rewriting lectures. For such purposes, and for the general interest of biologists when they have time, on holidays say, to read over all their science, these volumes ean be strongly recommended. They have good author and subject indices and the printing and production are excellent.

G. V. R. BORN

\section{DRUGS AND LIPIDS}

\section{Drugs affecting Lipid Metabolism}

Advances in Experimental Medicine and Biology, Vol. 4. (Proceedings of the Third International Symposium on Drugs affecting Lipid Metabolism, held in Milan, September $9-11,1968$.) Edited by William L. Holmes, Lars A. Carlson and Rudolfo Paoletti. Pp. xii +681 . (Plenum Press: New York, 1969.) \$27.50.

THE regulation of lipid metabolism by drugs and hormones is a relatively new study. For example, the mechanism of action of adrenaline in the control of the circulating level of free fatty acids (FFA) has been investigated only recently, although the effects of this hormone on carbohydrate metabolism have been documented for some time. Studies in this field have been stimulated mainly by the close interrelationships shown to exist between the development of atherosclerosis, the levels of circulating FFA. lipoproteins and cholesterol, and many of the papers are devoted to investigations along these lines.

The book contains fifty-two papers, most consisting of about ten pages, set out in the classical scientific paper style: introduction, results with reasonable experimental detail and discussion. Most papers are copiously illustrated with graphs, diagrams and tables. Some have photographs. Each paper is accompanied by a list of references, but surprisingly there are no reports of any discussions about the papers, other than the authors' own. even in a summarized form.

The report is divided into five sections and includes a final short section on methodology, mainly of lipid fractionation and analysis. This is a little removed from the main theme of the symposium, but it is nevertheless useful because progress in the field is often limited by the mothods of analysis available.

In the first section, "Drugs affecting FFA Mobilization", several of the drugs and many other factors such as cyclic AMP, ATP and prostaglandins which affect the levels of circulating FFA are discussed. Many papers emphasize the important relationships between cyclic AMP, adenyl cyclase and tissue lipases. The second section, which is the largest, entitled "Effects of Drugs on Plasma and Tissue Triglycerides", includes several papers dealing with clearing factor lipase and the mechanism of its release from tissues. Of special interest is a detailed account by M. ( $\therefore$ Schotz et al. on the morphology and metabolism of isolated fat cells which is illustrated by several good photomicrographs. The third section, "Drugs and Serum Lipoproteins", includes good reviews on "Typing of Hyperlipoproteinemias" and on the planning of clinical trials of new drugs such as 'Clofibrate' (Atromid-S) for its effect on ischaemic heart disease.
Cholesterol and bile acids form the main theme of the remaining section and this subject is considered from two main viewpoints: drugs which affect cholesterol biosynthesis and those which could affect its conversion to bile acids and subsequent excretion. This section includes a wide variety of subjects ranging from the long term appraisal of drugs such as oestrogens, nicotinic acid, dextrothyroxine and linoleamides on serum lipids and myocardial infaretion to factors affecting hydroxylation of cholesterol in the 7-position.

In all, the report covers a vast range of different approaches to the unsolved problems of lipid metabolism from fundamental biochemistry to clinical trials and, although the style of presentation by photographic reproduction will not appeal to all readers, nearly everyone interested in lipid metabolism will find much to interest thom.

E. D. WiLls

\section{IMMUNITY AGAINST PARASITES}

\section{The Immunology of Nematode Infections Trichinosis in Guinea-pigs as a Model \\ By David Catty. (Monographs in Allergy, Vol. 5.)} Pp. xiii + 134. (S. Karger: Basle and New York, 1969.) sFr/DM $39 ; \$ 9.35$.

IT is 28 years since Culbertson published his book Immunity against Animal Parasites, and interested parasitologists have long awaited the appearance of a new book on this subject. Unfortunately, they will surely be disappointed with this book. The extraordinarily worded title is misleading. The book is essentially Catty's PhD thesis on the immunity of guinea-pigs to Trichinella spiralis and has been presented in the style still used in some of these documents: plans, results and discussions of the experiments are dealt with in separate chapters rather than each experiment being treated as an entity. This makes the book difficult to read and even more difficult to abstract.

The introduction to the book gives a restricted review of the literature on the parasite. There are a number of unfortunate mistakes and a large body of literature on immunity against Trichinella is ignored; no references are made to the classic work of Culbertson and of Rappaport, to the interesting experiments of Matoff on the immune response of guinea-pigs, or to Jackson's beautiful immunofluorescence studies.

The results section is mainly taken up with Catty's observations on delayed hypersensitivity and various serologieal tests conducted in vitro and in vivo. Although these are of interest, some of the results depend on too few animals to be regarded as more than preliminary observations. This point is even more important in the consideration of the parasitological observations. Here the small groups, combined with the absence of statistical analyses, render the conclusions of dubious validity. The discussion and conclusion chapters are interesting but suffer from the restricted outlook of the whole book. The reference list contains a number of mistakes.

It is imperative that immunologists and parasitologists cooperate when working on the immunology of parasitic diseases, instead of isolating themselves in their individual disciplines. The fruits of such an interchange of ideas and techniques can be seen in the success of the Medical Research Council's parasitology group at the National Institute for Medical Research at Mill Hill; the dangers of isolation are illustrated by this book.

Publishers must be advised against publishing doctorate theses in this form. It would be better if they were broken down into separate papers which could be criticized by the appropriate editors and referees.

D. A. Denham 